\title{
A Segmented Attractor Network for Neuromorphic Associative Learning
}

\author{
Alexander Jones ${ }^{\dagger}$ \\ Department of Electrical \\ Engineering and Computer Science \\ University of Cincinnati \\ Cincinnati, OH 45219 USA \\ jones2a5@mail.uc.edu
}

\author{
Rashmi Jha \\ Department of Electrical \\ Engineering and Computer Science \\ University of Cincinnati \\ Cincinnati, OH 45219 USA \\ jhari@ucmail.uc.edu \\ Cory Merkel \\ Department of Computer \\ Engineering \\ Rochester Institute of Technology \\ Rochester, NY 14623 USA \\ cemeec@rit.edu
}

\author{
Ajey P. Jacob \\ Differentiating Technology \\ Research Group \\ GLOBALFOUNDRIES \\ Malta, NY 12020 USA \\ ajey.jacob@globalfoundries.com
}

\begin{abstract}
This work describes a segmented attractor network that records memories across different sets of information. Unlike typical attractor networks that can associate any given inputs with one another, the attractor network presented here tracks information across multiple sets where each set possesses one or more features. Hyperparameter analysis is performed on the network's recall capability by altering factors such as the network's size, the number of memories within the network, the amount of information required to perform recall, and the amount of feedback a synapse can provide. The analysis done shows an improved hit rate when increasing the features per set and allows the network to obtain a memory capacity higher than other standard attractor networks.
\end{abstract}

\section{CCS CONCEPTS}

- Computer systems organization $\rightarrow$ Architectures $\rightarrow$ Other architectures $\rightarrow$ Neural networks

\section{KEYWORDS}

Attractor network, associative learning, neuromorphic, simulation

\section{ACM Reference format:}

Alexander Jones, Rashmi Jha, Ajey P. Jacob and Cory Merkel. 2019. A Segmented Attractor Network for Neuromorphic Associative Learning. In Proceedings of International Conferences on Neuromorphic Systems (ICONS'19), July 23-25, 2019, Knoxville, TN, USA. ACM, New York, NY, USA, 8 pages.

Permission to make digital or hard copies of part or all of this work for personal or classroom use is granted without fee provided that copies are not made or distributed for profit or commercial advantage and that copies bear this notice and the full citation on the first page. Copyrights for components of this work owned by others than ACM must be honored. Abstracting with credit is permitted. To copy otherwise, or republish, to post on servers or to redistribute to lists, requires prior specific permission and/or a fee. Request permissions from Permissions@acm.org. ICONS'19, July 23-25, 2019, Knoxville, Tennessee, USA

(C) 2019 Association for Computing Machinery. 978-1-4503-7680-8/19/07...\$15.00 https://doi.org/10.1145/3354265.3354284

\section{INTRODUCTION}

The concept of associative learning has been studied for decades as a method of forming memories by creating correlations between information [1]. One of the most popular methods of implementing associative memory is the attractor network [2], which is a neural network that often stores memories in the form of connections between neurons [2-4]. Attractor networks are able to store information in the form of patterns of neuron outputs within the network either firing or not firing, or firing at different magnitudes [2-4]. Different sets of neurons firing simultaneously correspond to different memories within the network, and can be encoded to represent different forms of external stimulus. Different types of attractor networks including the Hopfield network [3], Storkey network [5], and others $[4,6-8]$ have been previously studied to understand these neuronal dynamics.

Many facets of attractor networks have been studied over the years, including the original implementation of the Hopfield net [3], the maximum capacity of networks [8], and other more applicationspecific studies [9-12]. One facet of attractor networks that must be studied in greater detail is their capability to recall memories across multiple sets of information where each set possesses one of many features it can exhibit at any given moment in time. This capability can be useful to perform tasks such as state estimation for systems possessing large arrays of sensor/analysis input to perform navigation or track a system's overall health. This paper proposes a novel architecture that standardizes sparsity for associative learning across multiple sets of information. The proposed design is suitable for both software and custom neuromorphic implementations. 


\section{ARCHITECTURE}

A basic form of the segmented attractor network architecture is shown in Figure 1. The objective of this architecture is to be able to associate various features belonging to different sets to one another. The network consists of the standard core components of a neural network and is similar in design to Hopfield networks [3]. The key difference in the design proposed here is how portions of the network are segmented.

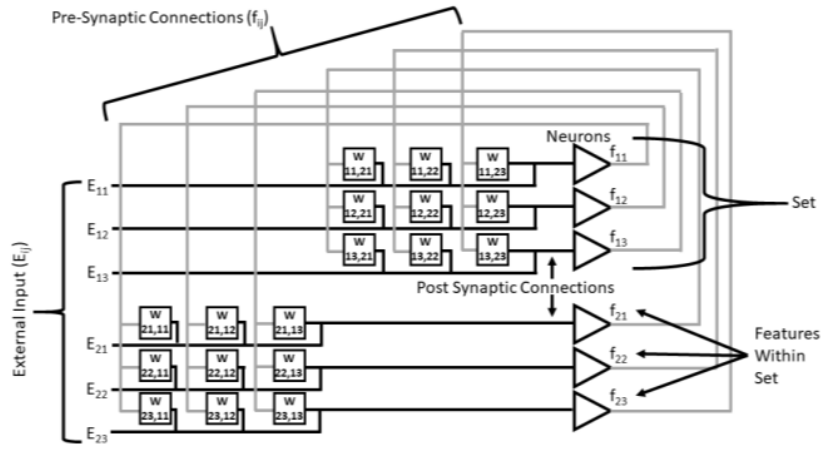

Figure 1: Basic example architecture of the segmented attractor network used in this work.

Within the architecture, the neurons are segmented into an array of sets defined as $s$ where the scalar magnitude of $s$ is defined as $y(t)$

$$
s=\left[\begin{array}{c}
s_{1} \\
s_{2} \\
\ldots \\
s_{n}
\end{array}\right]_{n \times 1}, \text { where }|s|=y(t)=\left[\begin{array}{c}
y_{1}(t) \\
y_{2}(t) \\
\ldots \\
y_{n}(t)
\end{array}\right]_{n \times 1}
$$

where $n$ represents the total number of sets within the network. In an application space, these sets can be used to define different types of incoming inputs such as sensor input for temperature, altitude, light intensity, air pressure, etc. Each sensing modality would belong to its own set. Each of these sets $\left(s_{i}\right)$ then possess an array of neurons that describe all features of the set. For example, the features of a set describing temperature could be each neuron within the set describing a certain temperature value $\left(10^{\circ} \mathrm{C}, 15^{\circ} \mathrm{C}\right.$, etc.). It is not necessary for each set to have the same number of features, nor is it necessary for the feature count of the sets to be the same as $n$. Any set within $s$ is defined as

$$
s_{i}=\left[\begin{array}{c}
f_{i 1} \\
f_{i 2} \\
\cdots \\
f_{i m}
\end{array}\right]_{m \times 1}, \text { where }\left|s_{i}\right|=y_{i}(t)=\left[\begin{array}{c}
y_{i 1}(t) \\
y_{i 2}(t) \\
\ldots \\
y_{i m}(t)
\end{array}\right]_{m \times 1}
$$

where $f_{i j}$ denotes an individual feature within the set. The indexing term, $i$, denotes the set to which $f_{i j}$ belongs, and therefore does not change from feature to feature within a set. The indexing term, $j$, differentiates each feature from one another within $s_{i}$. The term $m$ represents the total number of features within $s_{i}$. As mentioned before, $m$ does not have to be the same for each $s_{i}$. If $m$ is different for separate $s_{i}$, each $m$ can be indexed as $m_{i}$. For simplicity in equation definition in this work, it is assumed that all $m$ are equal. The architecture example shown in Figure 1 possesses two sets of neurons $(n=2)$ where each set has a feature count of three $(m=3)$. External sensory input is also provided to each feature within each set via an input line. The entire collection of external input provided to the network is defined by $E$

$$
E=\left[\begin{array}{c}
E_{1} \\
E_{2} \\
\ldots \\
E_{n}
\end{array}\right]_{n \times 1}
$$

where the external inputs are segmented into subsets $\left(E_{i}\right)$ that correspond to the same pattern of segmenting within $s$. Each individual set within $E$ can be defined as

$$
E_{i}=\left[\begin{array}{c}
E_{i 1} \\
E_{i 2} \\
\ldots \\
E_{i m}
\end{array}\right]_{m \times 1}
$$

where the indexing term $i$ once again indicates which set within $E$ each $E_{i j}$ belongs. For the architecture analyzed within this work, a one-hot encoding scheme was used for all $E_{i j}$ 's within a given $E_{i}$. This environmental rule was defined as it was assumed only one value could be provided to the network at any given time per $s_{i}$ since each $s_{i}$ could correspond to basic input values such as temperature, altitude, air pressure, etc. if the network was being used in an application space such as state estimation based upon a set of given input sensor data. This rule does not have to hold true for all usecases of the architecture, however. Within the artificial network analyzed here, $E$ can be provided to the system as a simple numerical input. If the system was to be implemented in a hardware-based neuromorphic architecture, $E$ could be provided to the system via a network of demultiplexers that receive their input from an array of sensors or other circuits analyzing the system's state.

Using these defined sets of features across all $s i$, recurrent connections can be made between features of each $s_{i}$ to form paths where associations between features can be made during use. These paths are defined as synapses $\left(w_{i k, j l}\right)$. Each synapse within the network belongs to one of the synapse blocks $\left(W_{i, j}\right)$ within the block matrix of synapses $W$

$$
W=\left[\begin{array}{ccc}
W_{1,1} & \cdots & W_{1, n} \\
\vdots & \ddots & \vdots \\
W_{n, 1} & \cdots & W_{n, n}
\end{array}\right]_{n \times n} \text {, where } W_{i, j}=0 \text { if } i=j
$$

where each $W_{i, j}$ within $W$ corresponds to a set of synapses that connects set $i$ with set $j$. A critical element of $W_{i, j}$ is the defined conditional where if $i=j$, all synapses within the $W_{i, j}$ matrix are forever fixed at 0 . This rule defines this network's "empty" diagonal across $s$, which is similar to how a traditional Hopfield network possesses an empty diagonal [3]. However, this network's empty diagonal exists across all locations where each $f_{i j}$ within a given $s_{i}$ could associate with one another. This architecture design choice allows any feature within a given $s_{i}$ to associate itself with any feature from another set, but not associate itself with any feature within $s_{i}$. This expanded empty diagonal helps define the 
A Segmented Attractor Network for Neuromorphic Associative Learning

segments within the network and distinguish each set from one another during memory formation and recall. Within any specific $W_{i, j}$, the collection of synapses can be further broken down into individual synapses

$$
W_{i, j}=\left[\begin{array}{ccc}
w_{i 1, j 1} & \cdots & w_{i 1, j m} \\
\vdots & \ddots & \vdots \\
w_{i m, j 1} & \cdots & w_{i m, j m}
\end{array}\right]_{m \times m}
$$

where $i k$ refers to the post synaptic feature connected to the synapse and $j l$ refers to the pre-synaptic feature connected to the synapse. With each $W_{i, j}$ being an $m$ by $m$ matrix of synapses, the total number of synapses within the network is defined by the equation

$$
N=n^{2} m^{2}
$$

where $N$ represents the total number of synapses within the network.

\section{MEMORY FORMATION AND RECALL}

\subsection{Memory Formation}

The first step in utilizing the segmented attractor network is populating its memory space with memories that correspond to relations between different values of $E_{i j}$ within each $E_{i}$ firing simultaneously. To form a basic memory within the architecture, a sensory input value is provided to two or more $E_{i j}$ 's where at least two $E_{i j}$ 's actively being provided input do not belong to the same $s i$. In terms of the generic network example being defined in this work, this input value is simply expressed as a value of one. This input of one along each $E_{i j}$ causes its corresponding feature, $f_{i j}$, to fire with a given output magnitude, $y_{i j}(t)$. In this defined network, $y_{i j}(t)$ simply shares a $1: 1$ relationship to the input being provided to $f_{i j}$. This relationship could be made nonlinear depending on the usecase defined for the network. Once $f_{i j}$ fires, any synaptic connections between features receiving both pre-synaptic and post synaptic stimulation simultaneously increases the weight of the synapse $\left(\uparrow w_{i k, j l}\right)$ from its initial value of zero to a non-zero value (wON). This change in the synapse's weight is defined as $\Delta w_{i k . j l}$ and follows the given rule

$$
\Delta w_{i k, j l}=\left\{\begin{aligned}
w_{O N}, & y_{j l}(t) * E_{i k} \geq 1 \\
0, & y_{j l}(t) * E_{i k}<1 \text { or } w_{i k, j l}=w_{O N}
\end{aligned}\right.
$$

where $y_{j l}(t)$ is the recurrent input to synapse $w_{i k, j l}$ shown in Figure 1. The rule dictates that if $y_{j l}(t)$ and $E_{i k}$ are both one simultaneously, $w_{i k, j l}$ is set to the value of $w_{O N}$. If $y_{j l}(t)$ or $E_{i k}$ are less than one or $w_{i k . j l}$ is already set to $w_{O N}, w_{i k . j l}$ will not change. This rule defines each $w_{i k . j l}$ as a one of two binary values: zero or $w_{O N}$. This ruleset could be modified depending on the network's application to instead use a non-binary synaptic weight value if needed where $w_{i k . j l}$ can assume multiple discrete values or a continuous analog range of values.
ICONS'19, July 23-25, 2019, Knoxville, TN USA

A visual example of a memory being formed within the defined example network is found in Figure 2. In Figure 2, the inputs $E_{12}$ and $E_{21}$ are both simultaneously being provided with input, which therefore increase the weights of synapses $w_{21,12}$ and $w_{12,21}$.

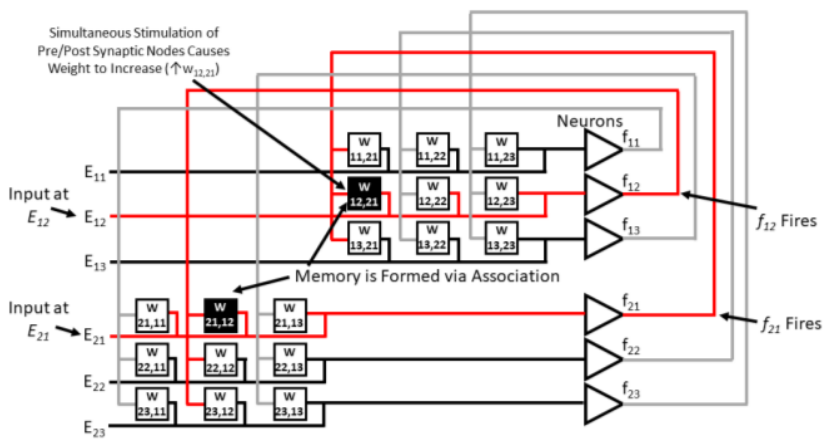

Figure 2: A demonstration of how a memory is formed within the example architecture to associate the two features $E_{12}$ and $\boldsymbol{E}_{21}$.

A final note on memory formation within the network is that over time not reversing any weights from a value of wON to zero could lead to an over saturation of the network. Inhibition can be used to counteract this over saturation, however forgetting memories was not a primary focus of this work. Therefore, no inhibition was used at any point during network evaluation.

\subsection{Memory Recall}

Once the segmented attractor network is populated with memories, the architecture should be able to perform recall. This network's definition of recall refers to giving input to one or more $E_{i j}$ 's that all belong to at least one memory already existing within the network. If all $E_{i j}$ values for a given memory are provided to the network, the network should in theory always provide a correct state estimation (unless the network has become over saturated). If not all $E_{i j}$ 's for a given memory are provided, the network must perform analysis to recall missing info.

The network follows an iterative process to determine its estimate for the correctly recalled state. To understand this process, $y(t)$ for the whole system must be defined and calculated. For all sets in the network, the output of all features is equal to

$$
y(t)=P+E
$$

where $P$ represents the post synaptic feedback provided to all features and $E$ still represents all external input provided to features. The term $P$ can be defined as

$$
P=W y(t-1)
$$

where $y(t-1)$ represents the output of all features at the previous iteration of the network $t-1 . W$ still represents all synaptic weights 
within the network. When Equation 9 is fully expanded (including expanding $W$ into its full $\mathrm{nm}$ by $\mathrm{nm}$ matrix form), one obtains

$$
\left[\begin{array}{c}
y_{11}(t) \\
y_{12}(t) \\
\cdots \\
y_{n m}(t)
\end{array}\right]=\left[\begin{array}{ccc}
w_{11,11} & \cdots & w_{11, n m} \\
\vdots & \ddots & \vdots \\
w_{n m, 11} & \cdots & w_{n m, n m}
\end{array}\right]\left[\begin{array}{c}
y_{11}(t-1) \\
y_{12}(t-1) \\
\cdots \\
y_{n m}(t-1)
\end{array}\right]+\left[\begin{array}{c}
E_{11} \\
E_{12} \\
\cdots \\
E_{n m}
\end{array}\right] \text { (11). }
$$

Once the magnitude for all $y_{i j}(t)$ has been found for given $E_{i j}$ 's to the network, the network attempts to look for a maximum $y_{i j}(t)$ within each $s_{i}$. If the network finds a $y_{i j}(t)$ within each $s_{i}$ that is larger than its counterparts, the network interprets that given set of maximum magnitude features as its state estimation. If any $s_{i}$ is determined to have no single maximum $y_{i j}(t)$, all values of $y(t)$ are applied to the presynaptic connections of $W$ and Equation 11 is calculated once again. This process continues until a maximum value for each $s_{i}$ is found or until it is clear no maximum value can be found for a given $s$. A diagram of a simple example of this process can be seen in Figure 3.

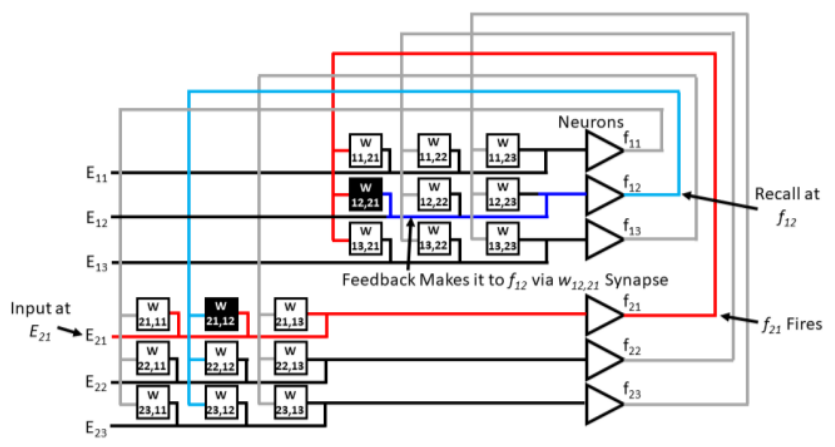

Figure 3: A demonstration on how memories are recalled within the example architecture by recalling the association between $E_{12}$ and $E_{21}$ by only giving input to $E_{21}$.

When multiple memories exist within a network, more complex situations can arise when attempting to perform recall. For example, if two memories exist within the example architecture where one associates $E_{11}$ and $E_{21}$ with one another and the other associates $E_{11}$ and $E_{22}$, if only $E_{21}$ receives input to the network (Figure 4), which of the two features should be chosen for the proper recall state? If there is a true state to be recalled in this case (i.e. there is a correct answer to the hidden feature set), there is a $50 \%$ chance the correct one is guessed in this case if it's guessed randomly. The network doesn't simply outright guess to determine the recalled state, however. It will indeed randomly pick one of the two tied maximum $y_{i j}(t)$ values from the given $s_{i}$, but it will perform one intermediary step prior to determining the recalled state. The randomly chosen $f_{i j}$ will have external input applied to it via its $E_{i j}$, and then the network will be reevaluated via Equation 11 to observe the maximum $y_{i j}(t)$ values once again. In the case of the example in Figure 4, this step really is not needed, but it is needed when performing state estimation on networks possessing higher amounts of sets where multiple memories exist. If the network could randomly guess between every set of tied $y_{i j}(t)$ values independently, it might accidentally guess a memory that was never formed in the network if reevaluation is not performed. This process of randomly selecting one of multiple tied $y_{i j}(t)$ is needed in software implementations when all initial weights are zero but might not be needed in non-zero weight initialization implementations where all initial weights are random. It might not be needed in hardware implementation as well due to device/circuit variability from synapse to synapse giving the synapses naturallyoccurring initial random weights.

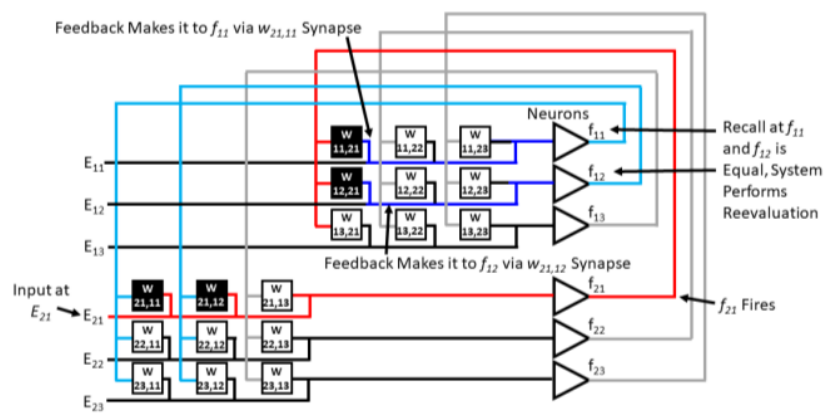

Figure 4: Example showing how the architecture needs to reevaluate at times when recalling memories and multiple features of the same set have similar magnitudes.

When performing analysis on larger networks with more memories within them, the architecture must be able to successfully recall despite having a higher complexity. Often, this requires the network to perform the iterative summation process of solving for the recalled state described previously. This process is akin to how other attractor networks converge to a normal answer by navigating through a set of network states $[2,4]$. In this architecture's case, the states are instead the value of each feature's output magnitude at the end of each iteration. A basic example of a network having to iterate multiple times can be seen in Figure 5.

In the example shown in Figure 5, the network has a total of three sets $(n=3)$ with two features per set. The value of woN for this network is set as 0.1 . In this network, three memories have already been created. The memories within the network are as follows:

\section{Memory \#1: $E_{11}, E_{21}, E_{32}$ are associated \\ Memory \#2: $E_{11}, E_{21}, E_{31}$ are associated \\ Memory \#3: $E_{11}, E_{22}, E_{31}$ are associated}

In Figure 5(a), the network is provided with input at $E_{11}$. The network's job is to recall the memory it is most likely currently observing based upon its input provided. Since $E_{11}$ is associated with all three memories within the network (along with other features such as $E_{21}$ and $E_{31}$ each belonging to multiple memories), it must perform multiple feedback iterations in the network to solve for the recalled state. Initially, the external input at $E_{11}$ causes the 
feature $f_{11}$ to have an output magnitude of one. This output is the only output to have a non-zero magnitude at this point in time. Others will receive input from feedback on the following iterations, however.

Once $f_{11}$ receives input and emits its output, its output then travels through all activated synapses with which it's connected (black synapses) and provides a feedback signal to any associated features (Figure 5(b)). In this case, that is $f_{21}, f_{22}, f_{31}$, and $f_{32}$. The magnitude of each synapse's feedback to its post synaptic neuron will be a product of wON and the magnitude of its pre-synaptic value, as dictated by Equation 11. For this iteration, the feedback provided by each activated synapse will be 0.1 , since $f_{11}$ has a magnitude of one and $w_{O N}=0.1$. Each of the features receiving feedback now have an output magnitude of 0.1. A final answer still hasn't been found yet, however. The network must iterate once again to find its solution.

On this iteration for the network, feedback will be calculated in accordance to the outputs that received a feedback signal on the previous iteration. $f_{21}, f_{22}, f_{31}$, and $f_{32}$ will provide feedback to neurons within the network via any connected, activated synapses (Figure 5(c)). Each of these features has a magnitude of 0.1 and will pass feedback to the network's features once again as a product of their magnitude and woN. On this iteration, each synapse will provide feedback of 0.01 since $y_{j l}(t) w_{i k, j l}=0.01$. Due to the configuration of activated synapses within the network, $f_{11}, f_{21}, f_{22}$, $f_{31}$, and $f_{32}$ will all receive feedback on this iteration of the network. $f_{11}$ will receive feedback of 0.04 , since it's receiving input from four activated synapses. $f_{21}$ and $f_{31}$ will both receive feedback of 0.02 since they both are connected to two activated synapses previously receiving feedback. $f_{22}$ and $f_{32}$ will only receive feedback of 0.01 since they are each only connected to a single activated neuron that is previously receiving feedback.

At this point in the analysis, three clear winning features (one per set) have emerged from the network. $f_{11}$ has a clear winning output amongst the first set since it was receiving external input. $f_{21}$ and $f_{31}$ have been evaluated as higher values from the other two sets within the network with respect to their counterparts $f_{22}$ and $f_{32}$. With this answer, the network has converged to the estimate of 11 , 21 , and 31 being the three features that it's observing given only external input for 11. This estimate corresponds to the second memory given to the network, which associated $E_{11}, E_{21}$, and $E_{31}$. This estimation is the most probabilistic solution from the network's perspective, as it has a $2 / 3$ chance of being correct if it guesses feature 21 from the features available within $s_{2}$ and a $2 / 3$ chance of being correct if it guesses feature 31 from the features in the $s_{3}$. Since both of these most probable estimates also happened to share a common memory, the system naturally estimated this memory to be the most likely answer. If those two features didn't share a common memory, the system would most likely have to perform an educated random guess as was shown in Figure 4 to find its final answer.

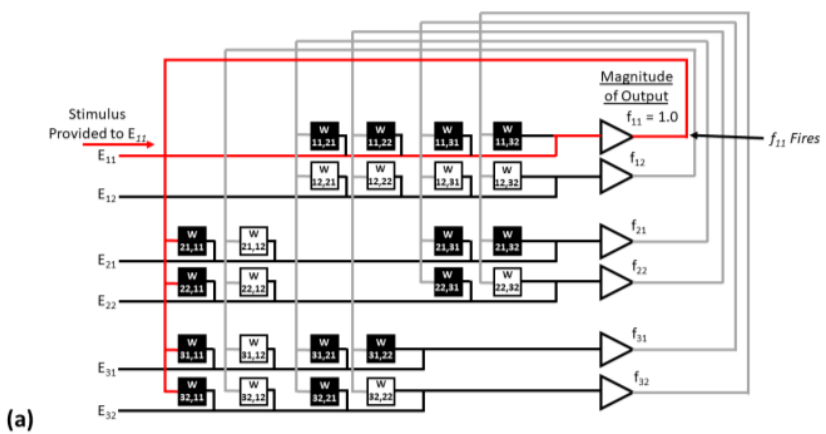

(b)

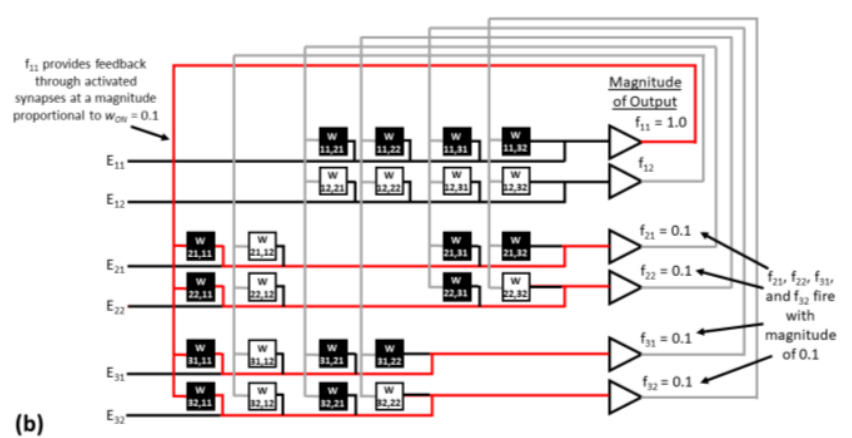

(c)

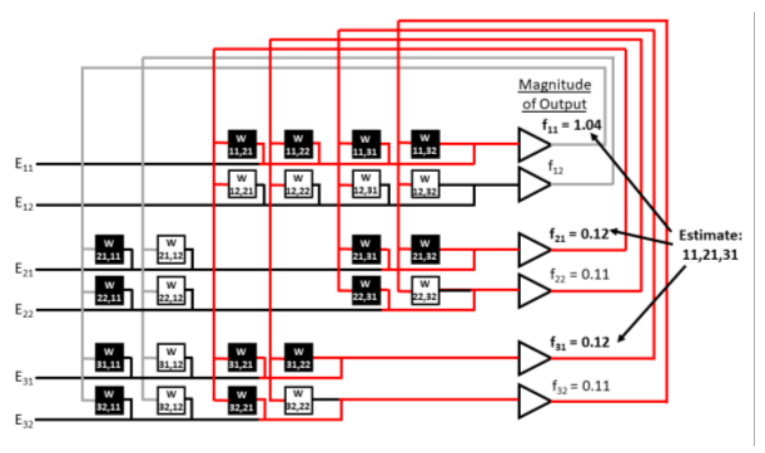

Figure 5: (a) A network with three pre-populated memories is provided stimulus along $E_{11}$. This stimulus causes $f_{11}$ to fire with a magnitude of 1 . (b) Feedback from $f_{11}$ propagates through any activated (black) synapses to other outputs within the network. This output is a product of the pre-synaptic output's magnitude and won. (c) A final iterative pass is performed on the network by using the feedback from the outputs that just received feedback previously and passing it once again through any activated pre-synaptic connections. With this iteration of the network, one output from each set of neurons has emerged as a maximum value. These maximum values act as the network's most probable state estimation in accordance to the input it initially received and the memories that already existed within the architecture's synaptic connections. 


\section{SIMULATION RESULTS AND DISCUSSION}

To simulate the attractor network, the simulation model was split into two parts: populating the network with memories and testing the network's recall capability once populated. Figure 6 portrays the full MATLAB simulation of the network from initialization of the network to analyzing the output during recall. To determine the hit rate during the second half of simulation, a random memory from the uniform distribution of memories across the network was chosen to be recalled. Before showing this memory to the network, a certain amount of non-zero $E_{i j}$ 's given to the network would be hidden from the network (defined as $I_{h i d}$ ). For example, if $n=10$ (therefore each memory associated ten $E_{i j}$ 's) and $I_{h i d}=1$, one of the ten $E_{i j}$ values from each memory would not be shown to the network. This is analogous to how networks such as the Hopfield network define the Hamming distance between network states [3]. The network must then properly recall what these hidden values are in the form of $\max (\mathrm{y}(\mathrm{t}))$. During simulation, many different hyperparameters were analyzed to observe the network's behavior including $n$ and $m$, memories within the network, won, and $I_{h i d}$.

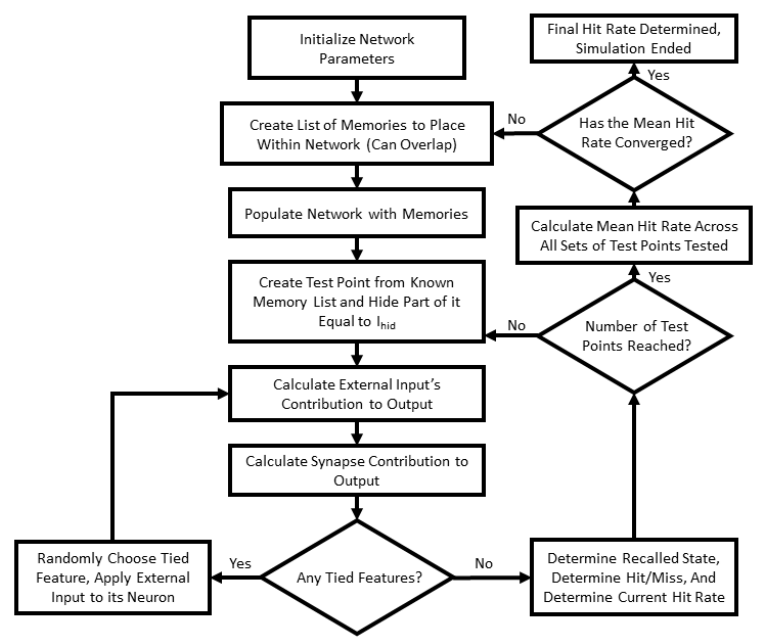

Figure 6: Algorithm used for analyzing the network. It should be noted that all work shown here uses a test point amount of 200 and convergence was detected to a change of 0.0001 .

The first analysis of the network looks at the network's hit rate as the network expands in size and the number of memories within the network increases. The results from this analysis can be seen in Figure 7. It should be noted that $I_{\text {hid }}$ and $w_{\text {on }}$ were fixed at 1 and 0.1 , respectively, for all simulations in Figure 7. In addition, for simplicity in displaying results, $n$ and $m$ were set to equal values. Naturally, as the network's size increases, its capacity for maintaining high hit rates on larger amounts of memories increases. This increase in capacity is primarily due to two factors. The first is due to memories having more space to stay separate from one another in larger networks. This extra space decreases the possibility that memories share features. A similar type of behavior has been seen in other studies [13]. The second is that $I_{\text {hid }}$ remains at a fixed value of one even for larger networks, which decreases the Hamming distance between the input and the original memory along the y-axis.
One feature of note within Figure 7 is the lighter blue tail that extends to higher memory counts from $n=m=6$ to $n=m=10$. The hit rate begins to increase for higher memory counts at this point, but then suddenly dies past 10 and 11 in the y-direction. The cause of this tail formation can be explained once one considers the value of $w_{O N}$ for all simulations shown in Figure 7. Once $n$ increases to $\sim 10-$ 11 , synapses can override external input to a neuron. If a $f_{i j}$ has a non-zero $E_{i j}$ (meaning that the system is certain that feature is the correct one), synapses to another $f_{i j}$ within that $f_{i j}$ 's $s_{i}$ can potentially override $E_{i j}$ to the correct feature within the set. This problem has immediate negative impact on the network's hit rate at higher memory counts. This characteristic means that the product of the network's set count (minus one) and woN is not allowed to exceed the magnitude of the external input provided to an individual feature. In other words,

$$
E>w_{O N}(n-1)
$$

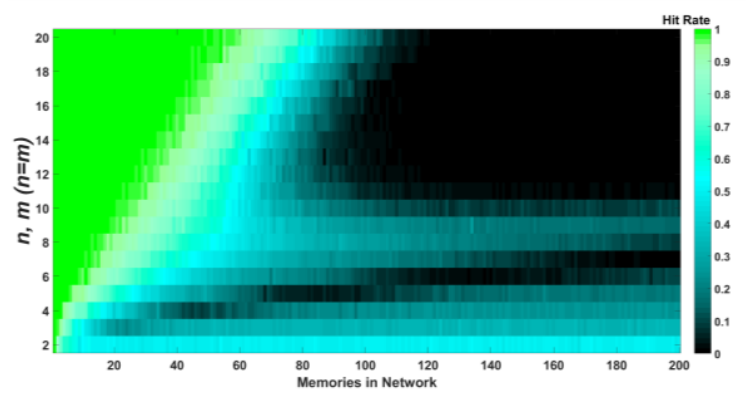

Figure 7: Colormap showing the average hit rate of the segmented attractor network as the number of sets/features and memories within the network are increased when $I_{\text {hid }}=1$ and won $=0.1$. $n=m$ for all networks.

If the value of woN is decreased to a value to ensure that Equation 12 holds true, the performance of the network for larger set/feature counts increases. This increase in performance can be seen in Figure 8. For the results in Figure 8, won was set to a value of 0.04 . If the set and feature count was further increased past 20, a similar dip in performance would be seen in the network's performance as is seen in Figure 7. If this network was ever implemented in hardware, this limitation shows the network's size is limited by the magnitude of feedback the synaptic circuit/device provides.

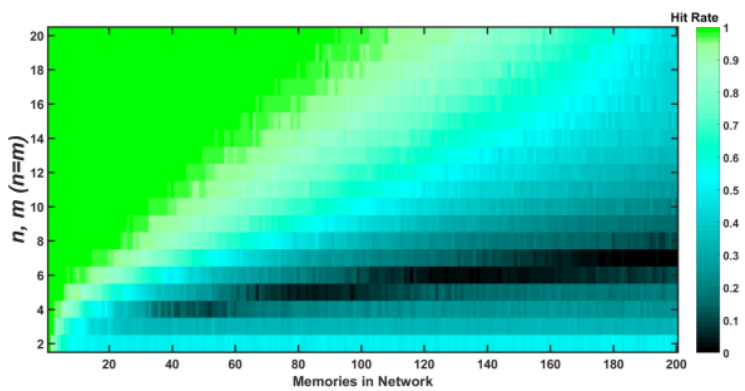

Figure 8: Colormap showing the average hit rate of the segmented attractor network as the number of sets/features and memories within the network are increased when $I_{\text {hid }}=1$ and $w o N=0.04 . \quad n=m$ for all networks. 
To expand on the study of $w_{O N}$, the size of the network can be fixed with woN increased to observe how it alters the network's hit rate. For this setup, a network of eight sets where each set contains eight features was chosen with $I_{\text {hid }}=1$. The value of wON $_{\text {ON }}$ was then increased from a value of 0.025 to 0.25 to observe how it affected hit rate. The results of this analysis are shown in Figure 9. A couple points can be drawn from what the figure shows. Firstly, at higher memory count values, a drop in the hit rate does occur past the point where $w_{O N}=0.125$, which is where Equation 12 predicts a drop in the hit rate would occur. Secondly, the overall hit rate appears to decrease even at lower values of woN. This observed behavior of the network requires a slight adjustment of Equation 12 if the desire is to maximize hit rate.

$$
E \gg w_{O N}(n-1)
$$

According to Figure 9, the difference between a single synapse's feedback input to the neuron versus the input applied by the external stimulus should be much larger than previously shown. Equation 13 and Figure 9 show that won should be made as small as feasibly possible when implementing this type of network.

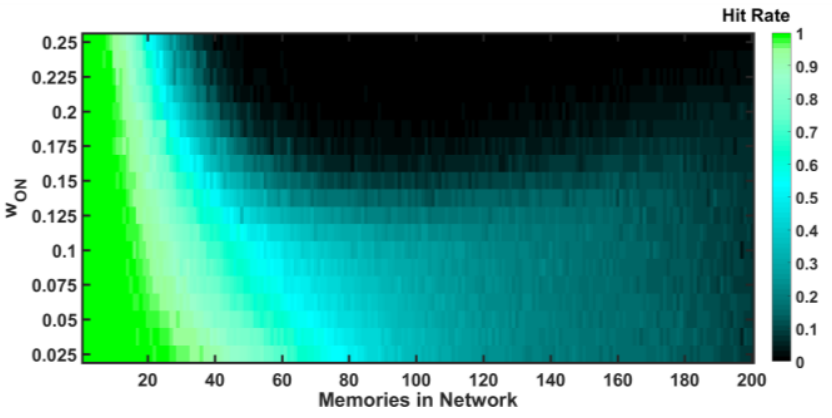

Figure 9: Colormap showing the average hit rate of an $n=8$, $m=8$ network where $I_{\text {hid }}=1$ as the memories within the network and $w o N$ are increased.

Feedback provided from synapses is not the only parameter that alters the network's hit rate. One of the primary parameters that affects hit rate is the amount of information that is hidden from the system (Ihid). The effect of increasing $I_{\text {hid }}$ was tested on a network with 20 sets of features where each set possessed 20 features. The value of wON was set to 0.04 to prevent feedback overriding external inputs. The results of $I_{\text {hid }}$ being varied from zero to 19 can be seen in Figure 10. As can be naturally expected, the network's performance decreases as $I_{\text {hid }}$ increases. High performance can still be attained at high values of $I_{\text {hid }}$ as long as the memory count within the network remains low. This performance disappears quickly when $I_{h i d}$ approaches its highest possible values, however.

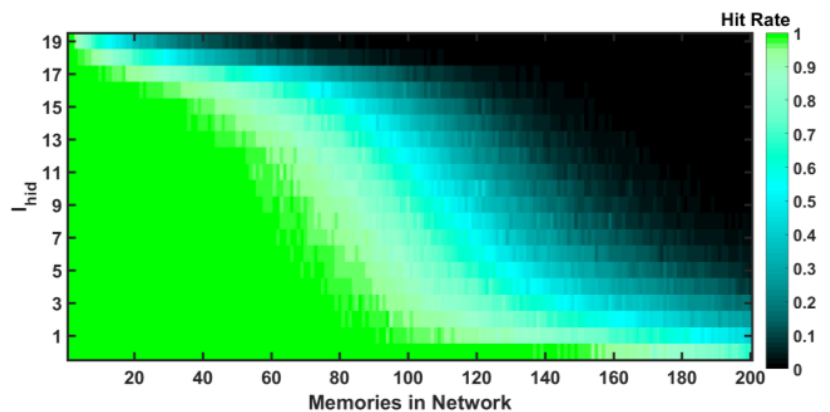

Figure 10: Colormap showing the average hit rate of a $n=20$, $\boldsymbol{m}=\mathbf{2 0}$ network as $I_{\text {hid }}$ and the number of memories within the network are increased. The value of won was set to 0.04 .

The final hyperparameters to analyze are $n$ and $m$. The values of $n$ and $m$ and their effect on the network were studied in two separate simulations. For the first simulation (Figure 11), $n$ was fixed to ten with $I_{\text {hid }}=5$. Values of $I_{\text {hid }}=5$ and $n=10$ sets the Hamming distance between memories and test points to the network to the value that other previously defined attractor networks use to evaluate memory capacity $[3,5,8]$. The value of $m$ was then increased from two to 40. As $m$ increases, the number of memories able to be perfectly recalled from within the network increases at a quicker pace than observed in Figure 7. According to Figure 11, a ten-set network with 40 features per set can store $\sim 140-150$ simultaneous memories near-perfectly. This increase in memory capacity is primarily due to the increased space within the feature space within each set. The increase in $m$ increases the likelihood that memories have separate features from one another if each memory within the network has equal probability of occurring (assuming uniform memory distribution). This result shows that given a high enough $m$, the network's memory capacity can surpass previously defined networks such as the Hopfield network $(0.12 n$ $0.15 n)[3,8,14]$ or Storkey's network $(0.3 n)[5,8]$, where $n$ is the number of neurons within the network [15]. For this particular set count (10), the network surpasses the capacity of the Hopfield and Storkey networks when $m$ reaches $\sim 15$ and $\sim 33$, respectively. At the highest feature pet set count in Figure 11, the memory capacity reaches $\sim 0.375 n$.

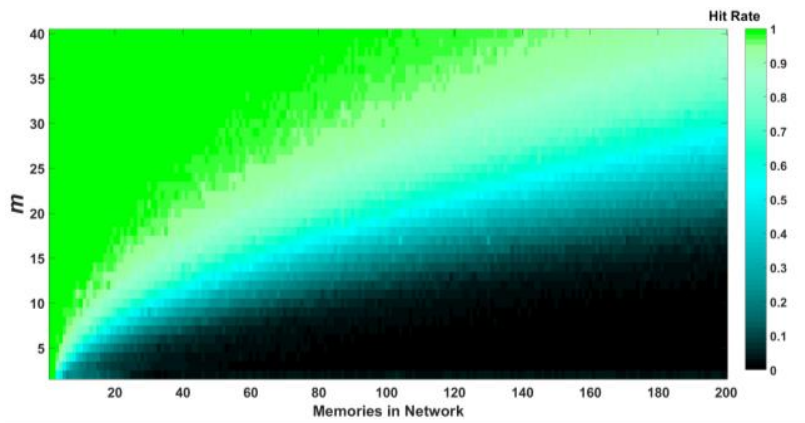

Figure 11: Colormap showing the average hit rate of the network as the features per set and memories within the network are increased. The values of $w_{o N}, I_{h i d}$, and $n$ were set to $0.01,5$, and 10 , respectively. 
If the set count within a network is changed in size while the feature count of each set remains fixed, different patterns in performance emerge from the network. In Figure 12, the number of features per set was fixed to a value of ten while the number of sets within the network was increased from two to 20 . The value of $I_{\text {hid }}$ remains at one. As the set count increases, the memory capacity of the network increases to an extent. Once the network reaches a set count of approximately seven, the capability of the network to performance perfect recall effectively stalls. Recall at lower probabilities does continue to increase until the set count reaches ten. After this point, the capability of recalling higher memory counts at lower probabilities begins to diminish. This performance decrease is likely due to the number of features within each set not increasing, which causes the amount of space the increasing amount of sets are having to share to become increasingly cramped. This smaller space increases the likelihood that memories share overlapping features, which can lead to incorrect recall and oversaturation of the network. The stagnation of perfect recall at higher set counts also likely contributes to the reason why the memory capacity of the largest network in Figure 8 (20 sets, 20 features per set) is less than the largest network in Figure 12. If this hypothesis is correct, it would also likely mean that the relationship between increasing the number of features per set and increasing the number of sets to maximize memory capacity is nonlinear.

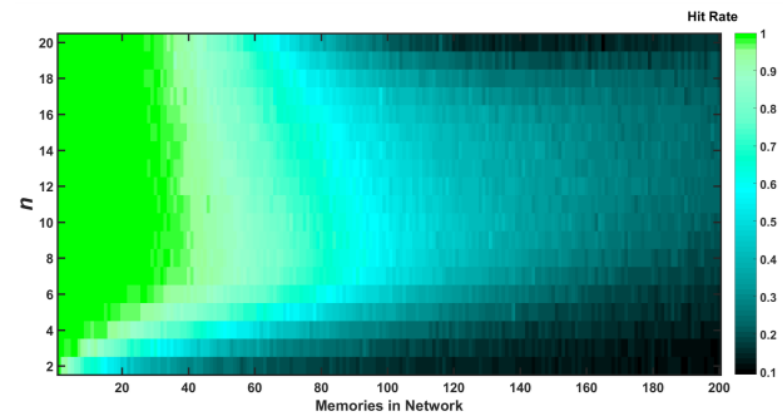

Figure 12: Colormap showing the average hit rate of the network as $\boldsymbol{n}$ and memories within the network are increased. The values of won, $I_{h i d}$, and $m$ are $0.04,1$, and 10, respectively.

\section{CONCLUSION}

This work analyzed a segmented attractor network that tracked associations between multiple sets of input where each set possessed its own set of features. A set of hyperparameters was altered to observe the architecture's hit rate and how it evolved with network size, number of memories within the system, the weight of synapses, and the amount of input hidden from the network during recall. The hyperparameter analysis revealed a list of behaviors that the network exhibits under different conditions. These behaviors are summarized in Table 1. These results show this type of attractor network is useful for any system that is using multiple types of input information to form associations between a large number of features. These associations can then be used to perform tasks such as state estimation when given partial input. If given enough features per set within the network, the network surpasses the memory capacity of other previously defined attractor networks.
Table 1: Summary of behaviors exhibited by the network studied.

\begin{tabular}{ll}
\hline Behavior & Source \\
\hline $\begin{array}{l}\text { The feedback provided from an individual } \\
\text { synapse }\left(w_{O N}\right) \text { caps memory capacity. }\end{array}$ & Figures 7-8 \\
\hline $\begin{array}{l}\text { Hit rate decreases if feedback from synapses can } \\
\text { exceed the input provided to neurons that }\end{array}$ & $\begin{array}{l}\text { Figures 7-9, } \\
\text { Equations } \\
\text { external input provides. }\end{array}$ \\
\hline $\begin{array}{l}\text { Increasing the amount of information hidden } \\
\text { from the network }\left(I_{\text {hid }}\right) \text { decreases hit rate. }\end{array}$ & Figure 10 \\
\hline Increasing $m$ increases hit rate. & Figure 11 \\
\hline $\begin{array}{l}\text { Given high enough } m \text {, the memory capacity of } \\
\text { the network surpasses both the Hopfield [3] and }\end{array}$ & \\
Storkey [5] networks. & \\
\hline $\begin{array}{l}\text { Hit rate saturates and diminishes at higher } \\
\text { memory counts if } m \text { is not increased while } n\end{array}$ & Figure 12 \\
increases. & \\
\hline $\begin{array}{l}\text { Hit rate saturates for near-perfect memory recall } \\
\text { if } m \text { is not increased while the set count }\end{array}$ & Figure 12 \\
increases.
\end{tabular}

\section{ACKNOWLEDGMENTS}

The authors would like to thank Clare Thiem at the Air Force Research Laboratory (AFRL) in Rome, NY, for his contributions to this work. This work is supported by the National Science Foundation award numbers: ECCS 1156294 and SHF-1718428.

\section{REFERENCES}

[1] D. J. Willshaw, O. P. Buneman, and H. C. Longuet-Higgins (1969). NonHolographic Associative Memory. Nature, 222, 960-962.

[2] D. J. Amit. 1989. Modeling Brain Function: The world of attractor neural networks. Cambridge University Press. Cambridge, United Kingdom.

[3] J. J. Hopfield (1982). Neural networks and physical systems with emergent collective computational abilities. Proc. Natl. Acad. Sci. USA, 79, 2554-2558.

[4] R. S. Zemel and M. C. Mozer (2001). Localist Attractor Networks. Neural Computation, 13, 1045-1064.

[5] A. Storkey (1997). Increasing the capacity of Hopfield network without sacrificing functionality. International Conference on Neural Networks, Houston, TX, USA.

[6] H. Lu (2001). Chaotic attractors in delayed neural networks. Physics Letters A, 298(2-3), 109-116.

[7] H. T. Siegelmann (2008). Analog-symbolic memory that tracks via reconsolidation. Physica D: Nonlinear Phenoma, 237 (9), 1207-1214.

[8] N. Davey and S. P. Hunt (1999). The capacity and attractor basins of associative memory models. International Work-Conference on Artificial Neural Networks, Alicante, Spain.

[9] V.-T. Pham et al. (2014). Hidden hyperchaotic attractor in a novel simple memristive neural network. Optoelectronics and Advanced Materials - Rapid Communications, 8 (11-12).

[10] R. Osan, A. B. L. Tort, and O. B. Amaral (2011). A Mismatch-Based Model for Memory Consolidation and Extinction in Attractor Networks. PLoS ONE, 6(8): e23113.

[11] A. M. Bruno, W. N. Frost, and M. D. Humphries (2017). A spiral attractor network drives rhythmic locomotion. eLife, 6: e27342.

[12] Z. Chen et al. (2017). Cracking the Cocktail Party Problem by Multi-beam Deep Attractor Network. 2017 IEEE Automatic Speech Recognition and Understanding Workshop, Okinawa, Japan.

[13] S. Romani et al. (2013). Scaling Laws of Associative Memory Retrieval. Neural Computation, 25(10), 2523-2544.

[14] E. Gardner (1987). Maximum Storage Capacity in Neural Networks. Europhys. Lett., 4 (4), 481-485.

[15] K. Gurney. 1997. An Introduction to Neural Networks. CRC Press. London, United Kingdom. 